Article

\title{
Exponential Synchronization of Two Complex Dynamical Networks of Random Disturbance with Both Mixed Coupled and Time-Varying Delay by Pinning Control
}

\section{Xuefei Wu}

School of Computer and Software Engineering, Shenzhen Polytechnic, Shenzhen 518055, China; E-Mail: wuxuefei@szpt.edu.cn; Tel.: +86-755-2673-1227.

Academic Editor: J. A. Tenreiro Machado

Received: 26 June 2015 / Accepted: 25 August 2015 / Published: 16 October 2015

\begin{abstract}
In this paper, the exponentially synchronization in the mean square is investigated for two different stochastic complex networks with hybrid coupling and time-varying delay via pinning control. By utilizing the Lyapunov stability theory, stochastic analysis theory, as well as matrix analysis, the sufficient conditions are derived to guarantee the exponential synchronization for any initial values through a feedback scheme. The numerical simulation is provided to show the effectiveness of the theoretical results.
\end{abstract}

Keywords: two complex dynamical networks; exponential synchronization in the mean square; random disturbance; pinning control

\section{Introduction}

Nowadays, the development of society is becoming more and more complicated and networked; thus, complex networks have emerged and aroused much attention [1]. A complex network is a structure that is made up of a large set of nodes (also called vertices, oscillators, agents) that are inter-connected to varying extents by a set of links (also called edges). Complex networks, indeed, are so ubiquitously found in nature and in the modern world that it is absolutely essential for us to have a thorough understanding of their dynamical behavior (for example, coupled biological systems, such as neural networks, and socially-interacting animal species [2]), and complex network synchronization holds particular promise for applications to many fields (for example, population dynamics [2,3], power systems [4,5] and automatic control [6]). 
Synchronization is a process in which two or more dynamical systems seek to adjust a certain prescribed property of their motion to a common behavior in the limit as time tends to infinity [7]. It is a phenomenon that has been widely investigated since it was discovered by Pecora and Carroll in 1990 [8]. Many synchronization patterns have been explored (for example, complete synchronization [9-11], cluster synchronization [12,13], phase synchronization [14], partial synchronization [15], projective synchronization [16,17]), and synchronization can be achieved by the use of pinning control [18,19], adaptive control [20-22], intermittent control [23], impulsive control [24,25], fuzzy control [26], hybrid control [27] or active control [28].

In the study of synchronization, inner synchronization is a synchronous phenomenon within a network and denotes the behavior of all of the nodes among one complex network becoming synchronous. Many different cases for inner synchronization have been investigated so far [4-28] On the contrary, outer synchronization $[29,30]$ is a synchronous phenomenon between networks, that is to say it refers to the synchronization occurring between two or more coupled complex networks regardless of the occurrence of inner synchronization, and many phenomena can be explained by outer synchronization in nature, such as an infectious disease spreading between different communities and different species' development in balance [30]. Li et al. first proposed the concept of outer synchronization and derived analytically a criterion for the synchronization of two networks that have the same topological connectivity in 2007 [30]. Additionally, outer synchronization between two coupled discrete-time networks that have the same connection topologies was derived analytically [31]. On the other hand, the problem of generalized outer synchronization between two completely different complex dynamical networks was investigated in [32]. The outer synchronization between two delay-coupled complex dynamical networks with nonidentical topological structures and a noise perturbation was regarded in [33]. However, there still exists the situation that the complex networks cannot achieve synchronization.

Stochastic perturbations and time delays are important considerations when simulating realistic complex networks, because signals traveling along real physical systems are usually randomly perturbed by the environmental elements [21]. In fact, signals transmitted between nodes of complex networks are unavoidably subject to stochastic perturbations from the environment, which may cause information contained in these signals to be lost [34]; the distance and finite speed of information transmission between pairs of nodes may cause time delays. Some results have recently appeared on the synchronization of complex networks with coupling delays [35-37] and stochastic perturbations [38-41].

Because of the complexity of complex networks, pinning control is a technique that applies controllers to only a small fraction of the nodes in a network, and the technique is important because it greatly reduces the number of controlled nodes for real-world complex networks (which, in most cases, is huge). In fact, pinning control can be so effective for some networks that a single pinning controller is required for synchronization; namely, for complex networks that have either a symmetric or an asymmetric coupling matrix [18]. Other pinning schemes, on the other hand, are capable of globally- and exponentially-stabilizing a network into a homogeneous state by using an optimal combination of the number of pinned nodes and the feedback control gain [19].

Motivated by the above discussions, in this paper, we study the mean square exponential synchronization of two nonidentical stochastic time-varying delayed complex networks by using the pinning control method. Some sufficient conditions are derived for mean square exponential 
synchronization of these networks by applying the Lyapunov-Krasovskii functional method. Our results will be applicable to many synchronization problems in different fields of science and technology.

The paper is organized as follows. In Section 2, a general model of two different stochastic complex networks with both time-varying delay dynamical nodes and time-varying delay coupling and some preliminaries are given. In Section 3, some exponential synchronization criteria for such complex dynamical networks are established. In Section 4, a numerical example for verifying the effectiveness of the theoretical results is provided. We conclude the paper in Section 5.

\section{Preliminaries}

\subsection{Notations}

Throughout this paper, $\mathbb{R}^{n}$ shall denote the $n$-dimensional Euclidean space and $\mathbb{R}^{n \times n}$ the set of all $n \times n$ real matrices. The superscript $T$ shall denote the transpose of a matrix or a vector; $\operatorname{Tr}(\cdot)$ denotes the trace of the corresponding matrix, $A^{S}=\left(A+A^{T}\right) / 2$ and $\mathbf{1}_{n}=(1,1, \ldots, 1)^{T} \in \mathbb{R}^{n}$; and $I_{n}$ denotes the $n$-dimensional identity matrix. For square matrices $M$, the notation $M>0$ (respectively, $<0$ ) shall mean that $M$ is a positive-definite (respectively, negative-definite) matrix, and $\lambda_{\max }(A)$ and $\lambda_{\min }(A)$ shall denote the greatest and least eigenvalues of a symmetric matrix, respectively. Let $\left(\Omega, \mathcal{F},\left\{\mathcal{F}_{t}\right\}_{t \geq 0}, \mathcal{P}\right)$ be a complete probability space with a filtration $\left\{\mathcal{F}_{t}\right\}_{t \geq 0}$ that is right continuous with $\mathcal{F}_{0}$ containing all of the $\mathcal{P}$-null sets. $C\left([-\tau, 0] ; \mathbb{R}^{n}\right)$ shall denote the family of continuous functions $\phi$ from $[-\tau, 0]$ to $\mathbb{R}^{n}$ with the uniform norm $\|\phi\|^{2}=\sup _{-\tau \leq s \leq 0} \phi(s)^{T} \phi(s)$ and $C_{\mathcal{F}_{0}}^{2}\left([-\tau, 0] ; \mathbb{R}^{n}\right)$ the family of all $\mathcal{F}_{0}$ measurable, $C\left([-\tau, 0] ; \mathbb{R}^{n}\right)$-valued stochastic variables $\xi=\{\xi(\theta):-\tau \leq \theta \leq 0\}$, such that $\int_{-\tau}^{0} \mathbb{E}|\xi(s)|^{2} d s \leq \infty$, where $\mathbb{E} \quad$ stands for the correspondent expectation operator with respect to the given probability measure $\mathcal{P}$.

\subsection{Network Model}

Consider a complex network consisting of $N$ identical linearly- and diffusively-coupled nodes with non-delayed and time-varying-delayed linear coupling. Additionally, every node in the network is an $n$-dimensional dynamical unit. Then, the network model of the drive system can be denoted as:

$$
\begin{aligned}
d y_{j}(t)= & \left\{f\left(t, y_{j}(t), y_{j}(t-\tau(t))\right)+\sum_{k=1}^{N} a_{j s}^{d} \Sigma y_{s}(t)+\sum_{k=1}^{N} b_{j s}^{d} \Sigma y_{s}\left(t-\tau_{c}(t)\right)\right\} d t \\
& +\sigma\left(t, y_{j}(t), y_{j}(t-\tau(t)), y_{j}\left(t-\tau_{c}(t)\right)\right) d w(t), \quad j=1,2, \ldots, N,
\end{aligned}
$$

where $y_{j}(t)=\left(y_{j 1}(t), y_{j 2}(t), \ldots, y_{j n}(t)\right)^{T} \in \mathbb{R}^{n}$ is the state vector of the $i$-th node of the network, $f\left(t, y_{j}(t), y_{j}(t-\tau(t))\right)=\left[f_{1}\left(t, y_{j}(t), y_{j}(t-\tau(t))\right), f_{2}\left(t, y_{j}(t), y_{j}(t-\tau(t))\right), \ldots, f_{n}\left(t, y_{j}(t)\right.\right.$, $\left.\left.y_{j}(t-\tau(t))\right)\right]^{T}$ is a continuous vector-valued function and $\Sigma=\operatorname{diag}\left(\varrho_{1}, \varrho_{2}, \ldots, \varrho_{n}\right)$ is an inner coupling of the networks that satisfies $\varrho_{k}>0, k=1,2, \ldots, n$. Here, $A^{d}=\left[a_{j s}^{d}\right] \in \mathbb{R}^{N \times N}$ and $B^{d}=\left[b_{j s}^{d}\right] \in \mathbb{R}^{N \times N}$ are the outer coupling matrices of the network at time $t$ and $t-\tau_{c}(t)$, respectively, such that $a_{j s}^{d} \geq 0$ for $i \neq k, a_{j j}^{d}=-\sum_{k=i, k \neq i}^{M} a_{j s}^{d}, b_{j s}^{d} \geq 0$ for $i \neq k$ and $b_{j j}^{d}=-\sum_{k=i, k \neq i}^{M} b_{j s}^{d} . \tau(t)$ is the inner time-varying delay satisfying $\tau \geq \tau(t) \geq 0$, and $\tau_{c}(t)$ is the coupling time-varying delay satisfying $\tau_{c} \geq \tau_{c}(t) \geq 0$. 
Finally, $\sigma\left(t, y_{i}(t), y_{j}(t-\tau(t)), y_{j}\left(t-\tau_{c}(t)\right)\right) \in \mathbb{R}^{n \times n}$, and $w(t)=\left(w_{1}(t), w_{2}(t), \ldots, w_{n}(t)\right)^{T} \in \mathbb{R}^{n}$ is a bounded vector-form Wiener process, satisfying:

$$
\mathbb{E} w_{k}(t)=0, \quad \mathbb{E} w_{k}^{2}(t)=1, \quad \mathbb{E} w_{k}(t) w_{k}(s)=0(s \neq t) .
$$

Compared to the drive system mentioned, the response complex network is denoted as consisting of $M$ identical linearly- and diffusively-coupled nodes with non-delayed and time-varying-delayed linear coupling. Additionally, every node in the network is an $n$-dimensional dynamical unit:

$$
\begin{aligned}
d x_{i}(t)= & \left\{f\left(t, x_{i}(t), x_{i}(t-\tau(t))\right)+\sum_{s=1}^{M} a_{i k}^{r} \Sigma x_{s}(t)+\sum_{s=1}^{M} b_{i k}^{r} \Sigma x_{s}\left(t-\tau_{c}(t)\right)\right\} d t \\
& +\sigma\left(t, x_{i}(t), x_{i}(t-\tau(t)), x_{i}\left(t-\tau_{c}(t)\right)\right) d w(t), \quad i=1,2, \ldots, M,
\end{aligned}
$$

where $x_{i}(t)=\left(x_{i 1}(t), x_{i 2}(t), \ldots, x_{i n}(t)\right)^{T} \in \mathbb{R}^{n} \quad$ is the state vector of the $j$-th node of the response network and $f\left(t, x_{i}(t), x_{i}(t-\tau(t))\right)=\left[f_{1}\left(t, x_{i}(t), x_{i}(t-\tau(t))\right), f_{2}\left(t, x_{i}(t), x_{i}(t-\tau(t))\right), \ldots, f_{n}\left(t, x_{i}(t)\right.\right.$, $\left.\left.x_{i}(t-\tau(t))\right)\right]^{T}$ is a continuous vector-valued function. Here, $A^{r}=\left[a_{i k}^{r}\right] \in \mathbb{R}^{M \times M}$ and $B^{r}=\left[b_{i k}^{r}\right] \in \mathbb{R}^{M \times M}$ are the outer coupling matrices of the network at time $t$ and $t-\tau_{c}(t)$, respectively, such that $a_{i k}^{r} \geq 0$ for $i \neq k, a_{i i}^{r}=-\sum_{k=i, k \neq i}^{M} a_{i k}^{r}, b_{i k}^{r} \geq 0$ for $i \neq k$ and $b_{i i}^{r}=-\sum_{k=i, k \neq i}^{M} b_{i k}^{r}$. In this paper, $A^{r}$ and $A^{d}$ are assumed to be irreducible in the sense that there are no isolated nodes.

The initial conditions associated with (2) are:

$$
y_{j}(s)=\xi_{j}^{d}(s), \quad x_{i}(s)=\xi_{i}^{r}(s), \quad-\check{\tau} \leq s \leq 0, \quad j=1,2, \ldots, N, \quad i=1,2, \ldots, M,
$$

where $\check{\tau}=\max \left\{\tau(t), \tau_{c}(t)\right\}, \xi_{j}^{d}, \xi_{i}^{r} \in C_{\mathcal{F}_{0}}^{b}\left([-\check{\tau}, 0], \mathbb{R}^{n}\right)$ with the norm $\|\xi\|^{2}=\sup _{-\check{\tau} \leq s \leq 0} \xi(s)^{T} \xi(s)$.

\subsection{Some Definitions, Lemmas and Assumptions}

In the following, we present some definitions and lemmas that will be required throughout this paper.

Definition 1. The drive network (2) and the response network (1) are said to be exponentially synchronized in the mean square if the trivial solutions of Systems (2) and (1) are such that:

$$
\sum_{i=1}^{M} \sum_{j=1}^{N} \mathbb{E}\left\|x_{i}\left(t, t_{0}, \xi_{i}^{r}\right)-y_{j}\left(t, t_{0}, \xi_{j}^{d}\right)\right\|^{2} \leq K e^{-\kappa t}
$$

for some $K>0$ and some $\kappa>0$ for any initial data $\xi_{i}^{r}, \xi_{j}^{d} \in \mathcal{C}_{\mathcal{F}_{0}}^{b}\left([-\hat{\tau}, 0] ; \mathbb{R}^{n}\right)$.

Definition 2. [21] A continuous function $f(t, x, y):[0,+\infty] \times \mathbb{R}^{n} \times \mathbb{R}^{n} \rightarrow \mathbb{R}^{n}$ is said to belong to the function class QUAD, denoted by $f \in \operatorname{QUAD}(P, \Delta, \eta, \zeta)$ for some given matrix $\Sigma=\operatorname{diag}\left\{\varrho_{1}, \varrho_{2}, \ldots, \varrho_{n}\right\}$, if there exists a positive definite diagonal matrix $P=\operatorname{diag}\left\{p_{1}, p_{2}, \ldots, p_{n}\right\}$, a diagonal matrix $\Delta=\operatorname{diag}\left\{\delta_{1}, \delta_{2}, \ldots, \delta_{n}\right\}$ and constants $\eta>0, \zeta>0$, such that $f(\cdot)$ satisfies the condition:

$$
(x-y)^{T} P((f(t, x, z)-f(t, y, w))-\Delta \Sigma(x-y)) \leq-\eta(x-y)^{T}(x-y)+\zeta(z-w)^{T}(z-w)
$$

for all $x, y, z, w \in \mathbb{R}^{n}$. 
Almost all of the well-known chaotic systems belong to the function class QUAD, such as the Lorenz system, the Rössler system, the Chen system, the delayed Chua's circuit, the delayed Hopfield neural network and the logistic delayed differential system [21,23].

Lemma 1. Consider an $n$-dimensional stochastic differential equation:

$$
d x(t)=f(t, x(t), x(t-\tau)) d t+\sigma(t, x(t), x(t-\tau))) d \omega(t)
$$

Let $C^{2,1}\left(\mathbb{R}_{+} \times \mathbb{R}^{n} ; \mathbb{R}_{+}\right)$denote the family of all nonnegative functions $V(t, x)$ on $\mathbb{R}_{+} \times \mathbb{R}^{n}$, which are twice continuously differentiable in $x$ and once differentiable in $t$. If $V \in C^{2,1}\left(\mathbb{R}_{+} \times \mathbb{R}^{n} ; \mathbb{R}_{+}\right)$, define an operator $\mathcal{L} V$ form $\mathbb{R}_{+} \times \mathbb{R}^{n}$ to $\mathbb{R}$ by:

$$
\mathcal{L} V(t, x)=V_{t}(t, x)+V_{x}(t, x) f(t, x, y)+\frac{1}{2} \operatorname{Tr}\left[\sigma(t, x, y)^{T} V_{x x} \sigma(t, x, y)\right],
$$

where $V_{t}(t, x)=\partial V(t, x) / \partial t, V_{x}(t, x)=\left(\partial V(t, x) / \partial x_{1}, \ldots, \partial V(t, x) / \partial x_{n}\right), V_{x x}(t, x)=\left(\frac{\partial^{2} V(t, x)}{\partial x_{i} x_{j}}\right)_{n \times n}$. If $V \in C^{2,1}\left(\mathbb{R}_{+} \times \mathbb{R}^{n} ; \mathbb{R}_{+}\right)$, then for any $\infty>t>t_{0} \geq 0$,

$$
\mathbb{E} V(t, x(t))=\mathbb{E} V\left(t_{0}, x\left(t_{0}\right)\right)+\mathbb{E} \int_{t_{0}}^{t} \mathcal{L} V(s, x(s)) d s
$$

as long as the expectations of the integrals exist $[42,43]$.

The following assumptions will be used throughout this paper for establishing the synchronization conditions.

H1 $\tau(t)$ and $\tau_{c}(t)$ are bounded and continuously differentiable functions, such that $0<\tau(t) \leq \tau$, $\dot{\tau}(t)<\bar{\tau}, 0<\tau_{c}(t) \leq \tau_{c}$ and $\dot{\tau}_{c}(t)<\bar{\tau}_{c}$. Let $\check{\bar{\tau}}=\max \left\{\bar{\tau}, \bar{\tau}_{c}\right\}$.

H2 Let $\sigma_{i j}\left(t, e_{i j}(t), e_{i j}(t-\tau(t)), e_{i j}\left(t-\tau_{c}(t)\right)\right)=\sigma\left(t, x_{i}(t), x_{i}(t-\tau(t)), x_{i}\left(t-\tau_{c}(t)\right)\right)-\sigma\left(t, y_{j}(t)\right.$, $\left.y_{j}(t-\tau(t)), y_{j}\left(t-\tau_{c}(t)\right)\right)$. Then, there exist positive definite constant matrices $\Upsilon_{i j}^{1}, \Upsilon_{i j}^{2}$ and $\Upsilon_{i j}^{3}$ for $i=1,2, \ldots, M$ and $j=1,2, \ldots, N$, such that:

$$
\begin{aligned}
& \operatorname{Tr}\left[\sigma_{i j}\left(t, e_{i j}(t), e_{i j}(t-\tau(t)), e_{i j}\left(t-\tau_{c}(t)\right)\right)^{T} P \sigma_{i j}\left(t, e_{i j}(t), e_{i j}(t-\tau(t)), e_{i j}\left(t-\tau_{c}(t)\right)\right)\right] \\
\leq & e_{i j}(t)^{T} \Upsilon_{i j}^{1} e_{i j}(t)+e_{i j}(t-\tau(t))^{T} \Upsilon_{i j}^{2} e_{i j}(t-\tau(t))+e_{i j}\left(t-\tau_{c}(t)\right)^{T} \Upsilon_{i j}^{3} e_{i j}\left(t-\tau_{c}(t)\right),
\end{aligned}
$$

where $e_{i j}(t)=x_{i}(t)-y_{j}(t)$.

Remark 1. The assumptions are common in the literature on stochastic complex networks with time-varying delay, such as [21,23,27,38-40]. Assumption H1 restricts the time delay, and Assumption $\mathrm{H} 2$ restricts the noise strength. We shall simply write:

$$
\begin{aligned}
& \Upsilon^{1}=\operatorname{diag}\left\{\Upsilon_{11}^{1}, \Upsilon_{21}^{1}, \ldots, \Upsilon_{M 1}^{1}, \Upsilon_{12}^{1}, \ldots, \Upsilon_{M 2}^{1}, \ldots, \Upsilon_{1 N}^{1}, \ldots, \Upsilon_{M N}^{1}\right\} \\
& \Upsilon^{2}=\operatorname{diag}\left\{\Upsilon_{11}^{2}, \Upsilon_{21}^{2}, \ldots, \Upsilon_{M 1}^{2}, \Upsilon_{12}^{2}, \ldots, \Upsilon_{M 2}^{2}, \ldots, \Upsilon_{1 N}^{2}, \ldots, \Upsilon_{M N}^{2}\right\} \\
& \Upsilon^{3}=\operatorname{diag}\left\{\Upsilon_{11}^{3}, \Upsilon_{21}^{3}, \ldots, \Upsilon_{M 1}^{3}, \Upsilon_{12}^{3}, \ldots, \Upsilon_{M 2}^{3}, \ldots, \Upsilon_{1 N}^{3}, \ldots, \Upsilon_{M N}^{3}\right\}
\end{aligned}
$$




\section{Main Result}

In the paper, the synchronization between the drive network (1) and the response network (2) will be investigated. In order to reach the synchronization, some extra controllers are required to add on the partial nodes of the response system. Without loss of generality, let the first $l$ nodes in the response system be controlled. Additionally, the response network with a control scheme is given by:

$$
\begin{aligned}
d x_{i}(t)= & \left\{f\left(t, x_{i}(t), x_{i}(t-\tau(t))\right)+\sum_{k=1}^{M} a_{i k}^{r} \Sigma x_{k}(t)+\sum_{k=1}^{M} b_{i k}^{r} \Sigma x_{k}\left(t-\tau_{c}(t)\right)+u_{i}(t)\right\} d t \\
& +\sigma_{i}\left(t, x_{i}(t), x_{i}(t-\tau(t)), x_{i}\left(t-\tau_{c}(t)\right)\right) d w(t), \quad i=1,2, \ldots, M,
\end{aligned}
$$

where $u_{i}(t)(i=1,2, \ldots, M)$ are the linear state feedback controllers that are defined by:

$$
u_{i}(t)= \begin{cases}-\varepsilon_{i} \Sigma\left(x_{i}(t)-y_{1}(t)\right), & i=1,2, \ldots, l, \\ 0, & i=l+1, l+2, \ldots, M,\end{cases}
$$

where $\varepsilon_{i}>0(i=1,2, \ldots, l)$ are the control gains, denoted by $\Xi=\operatorname{diag}\left\{\varepsilon_{1}, \varepsilon_{2}, \ldots, \varepsilon_{l}, 0, \ldots, 0\right\} \in$ $\mathbb{R}^{M \times M}$. Define $e_{i j}(t)=x_{i}(t)-y_{j}(t)(i=1,2, \ldots, M, j=1,2, \ldots, N)$ as the synchronization error. Then, according to the controller (7), the error system is:

$$
\begin{aligned}
d e_{i j}(t)= & \left\{f\left(t, x_{i}(t), x_{i}(t-\tau(t))\right)-f\left(t, y_{j}(t), y_{j}(t-\tau(t))\right)+\sum_{k=1}^{M} a_{i k}^{r} \Sigma x_{k}(t)\right. \\
& \left.\left.-\sum_{s=1}^{N} a_{j s}^{d} \Sigma y_{s}(t)+\sum_{k=1}^{M} b_{i k}^{r} \Sigma x_{k}\left(t-\tau_{c}(t)\right)-\sum_{s=1}^{N} b_{j s}^{d} y_{s}\left(t-\tau_{c}(t)\right)\right)+u_{i}(t)\right\} d t \\
& +\left\{\sigma_{i}\left(t, x_{i}(t), x_{i}(t-\tau(t)), x_{i}\left(t-\tau_{c}(t)\right)\right)-\sigma_{j}\left(t, y_{j}(t), y_{j}(t-\tau(t)), y_{j}\left(t-\tau_{c}(t)\right)\right)\right\} d w(t), \\
& i=1, \ldots, M, j=1, \ldots, N .
\end{aligned}
$$

Remark 2. The pinning controllers (7) are added to a part of the node in response networks by the information of the first node in drive networks, which is easy to achieve. The drive networks (1) and response networks (2) reach outer synchronization in the mean square by pinning controllers (7) as long as error System (8) is globally and exponentially stable in the mean square.

We present the outer synchronization criteria for two stochastic complex networks with time-varying delay.

Theorem 1. Let Assumptions $\mathrm{H} 1$ and $\mathrm{H} 2$ be true, and let $f \in \operatorname{QUAD}(P, \Delta, \eta, \zeta)$. If there exist $\alpha, \beta$, $\tau(t)$ and $\tau_{c}(t)$, such that:

$$
0 \leq \check{\bar{\tau}} \leq 1-\frac{b+c}{a}, \quad \text { and } \quad a>0
$$

where:

$$
\begin{aligned}
& a=\lambda_{\min }\left\{(\eta-\alpha-\beta) I_{M N n}-I_{M} \otimes I_{N} \otimes(\Delta \Sigma)\right. \\
& \left.-\left(A^{d} \otimes I_{M}+I_{N} \otimes A^{r}-\Xi \otimes I_{N}^{1}\right)^{S} \otimes(P \Sigma)-\frac{1}{2} \Upsilon_{1}\right\}, \\
& b=\lambda_{\max }\left\{\zeta I_{M N n}+\frac{1}{2} \Upsilon_{2}\right\}, \\
& c=\lambda_{\max }\left\{\left(\frac{1}{\beta}\left(B^{d}\right)^{T}\left(B^{d}\right) \otimes I_{M}+\frac{1}{\alpha} I_{N} \otimes\left(B^{r}\right)^{T}\left(B^{r}\right)\right) \otimes(P \Sigma)^{T}(P \Sigma)+\frac{1}{2} \Upsilon_{3}\right\},
\end{aligned}
$$


then the solutions $e_{i j}(t)(i=1,2, \ldots, M, j=1,2, \ldots, N)$ of System (8) are globally and exponentially stable in the mean square.

Proof. Define the Lyapunov-Krasovskii function:

$$
V(t, e(t))=\frac{1}{2} \sum_{i=1}^{M} \sum_{j=1}^{N} e_{i j}(t)^{T} P e_{i j}(t) .
$$

By Lemma 1, we have:

$$
\begin{aligned}
\mathcal{L} V(t, e(t), r)= & \sum_{i=1}^{M} \sum_{j=1}^{N} e_{i j}(t)^{T} P\left\{f\left(t, x_{i}(t), x_{i}(t-\tau(t))\right)-f\left(t, y_{j}(t), y_{j}(t-\tau(t))\right)\right. \\
& +\sum_{k=1}^{M} a_{i k}^{r} \Sigma x_{k}(t)-\sum_{s=1}^{N} a_{j s}^{d} \Sigma y_{s}(t) \\
& \left.\left.+\sum_{k=1}^{M} b_{i k}^{r} \Sigma x_{k}\left(t-\tau_{c}(t)\right)-\sum_{s=1}^{N} b_{j s}^{d} y_{s}\left(t-\tau_{c}(t)\right)\right)+u_{i}(t)\right\} \\
& +\frac{1}{2} \sum_{i=1}^{M} \sum_{j=1}^{N} \operatorname{Tr}\left\{\sigma_{i j}\left(t, e_{i j}(t), e_{i j}(t-\tau(t)), e_{i j}\left(t-\tau_{c}(t)\right)\right)^{T}\right. \\
& \left.\times P \sigma_{i j}\left(t, e_{i j}(t), e_{i j}(t-\tau(t)), e_{i j}\left(t-\tau_{c}(t)\right)\right)\right\}
\end{aligned}
$$

For simplicity, denote $e_{\cdot j}(t)=\left[e_{1 j}^{T}(t), e_{2 j}^{T}(t), \ldots, e_{M j}^{T}(t)\right]^{T} \in \mathbb{R}^{n M} ; \quad e_{i \cdot}(t)=$ $\left[e_{i 1}^{T}(t), e_{i 2}^{T}(t), \ldots, e_{i N}^{T}(t)\right]^{T} \in \mathbb{R}^{n N} ; e(t)=\left[e_{\cdot 1}^{T}(t), e_{\cdot 2}^{T}(t), \ldots, e_{\cdot N}^{T}(t)\right]^{T} \in \mathbb{R}^{M N n}$.

Because $f \in \operatorname{QUAD}(P, \Delta, \eta, \zeta)$, we get:

$$
\begin{aligned}
& \sum_{i=1}^{M} \sum_{j=1}^{N} e_{i j}(t)^{T} P\left\{f\left(t, x_{i}(t), x_{i}(t-\tau(t))\right)-f\left(t, y_{j}(t), y_{j}(t-\tau(t))\right)\right\} \\
& \quad \leq-\eta e^{T}(t) e(t)+\zeta e^{T}(t-\tau(t)) e(t-\tau(t))+e^{T}(t) I_{M} \otimes I_{N} \otimes(P \Delta \Sigma) e(t)
\end{aligned}
$$

From the definitions of $A^{r}\left(\sum_{k=i}^{M} a_{i k}^{r}=0\right)$, the following equation holds:

$$
\begin{gathered}
\sum_{i=1}^{M} \sum_{j=1}^{N} e_{i j}^{T}(t) P \sum_{k=1}^{M} a_{i k}^{r} \Sigma x_{k}(t)=\sum_{i=1}^{M} \sum_{j=1}^{N} e_{i j} P \sum_{k=1}^{M} a_{i k}^{r} \Sigma\left(x_{k}(t)-y_{j}(t)\right) \\
=\sum_{j=1}^{N} e_{\cdot j}^{T}(t) A^{r} \otimes(P \Sigma) e_{\cdot j}(t)=e^{T}(t) I_{N} \otimes A^{r} \otimes(P \Sigma) e(t)
\end{gathered}
$$

Similarly,

$$
\begin{gathered}
\sum_{i=1}^{M} \sum_{j=1}^{N} e_{i j}^{T}(t) P \sum_{k=1}^{M} b_{i k}^{r} \Sigma x_{k}\left(t-\tau_{c}(t)\right)=e^{T}(t) I_{N} \otimes B^{r} \otimes(P \Sigma) e\left(t-\tau_{c}(t)\right), \\
\sum_{i=1}^{M} \sum_{j=1}^{N} e_{i j}^{T}(t) P \sum_{s=1}^{N} a_{j k}^{d} \Sigma x_{s}(t)=e^{T}(t) A^{d} \otimes I_{M} \otimes(P \Sigma) e(t), \\
\sum_{i=1}^{M} \sum_{j=1}^{N} e_{i j}^{T}(t) P \sum_{s=1}^{N} b_{j k}^{d} \Sigma x_{s}\left(t-\tau_{c}(t)\right)=e^{T}(t) B^{d} \otimes I_{M} \otimes(P \Sigma) e\left(t-\tau_{c}(t)\right) .
\end{gathered}
$$


By using $x^{T} y \leq c x^{T} x+\frac{1}{c} y^{T} y$ for any $x, y \in R^{N}$ and $c>0$, we can obtain:

$$
\begin{aligned}
& e^{T}(t) I_{N} \otimes B^{r} \otimes(P \Sigma) e\left(t-\tau_{c}(t)\right) \\
\leq & \alpha e^{T}(t) e(t)+\frac{1}{\alpha} e\left(t-\tau_{c}(t)\right) I_{N} \otimes\left(B^{r}\right)^{T} B^{r} \otimes(P \Sigma)^{T}(P \Sigma) e\left(t-\tau_{c}(t)\right)
\end{aligned}
$$

and:

$$
\begin{aligned}
& e^{T}(t) B^{d} \otimes I_{M} \otimes(P \Sigma) e\left(t-\tau_{c}(t)\right) \\
\leq & \beta e^{T}(t) e(t)+\frac{1}{\beta} e^{T}\left(t-\tau_{c}(t)\right)\left(\left(B^{d}\right)^{T} B^{d} \otimes I_{M} \otimes(P \Sigma)^{T}(P \Sigma)\right) e\left(t-\tau_{c}(t)\right)
\end{aligned}
$$

where $\alpha>0$ and $\beta>0$.

Denote $I_{N}^{1}=\operatorname{diag}\{1,0, \ldots, 0\} \in \mathbb{R}^{N \times N}$; we have:

$$
\sum_{i=1}^{l} \sum_{j=1}^{N} e_{i j}^{T}(t) P u_{i}(t)=-\sum_{i=1}^{l} \sum_{j=1}^{N} e_{i j}^{T}(t) P \varepsilon_{i} \Sigma e_{i 1}(t)=-e^{T}(t) \Xi \otimes I_{N}^{1} \otimes(P \Sigma) e(t) .
$$

Using Assumption H2, we get:

$$
\begin{aligned}
& \sum_{i=1}^{M} \sum_{j=1}^{N} \operatorname{Tr}\left\{\sigma_{i j}\left(t, e_{i j}(t), e_{i j}(t-\tau(t)), e_{i j}\left(t-\tau_{c}(t)\right)\right)^{T}\right. \\
& \left.\times P \sigma_{i j}\left(t, e_{i j}(t), e_{i j}(t-\tau(t)), e_{i j}\left(t-\tau_{c}(t)\right)\right)\right\} \\
\leq & \sum_{i=1}^{M} \sum_{j=1}^{N}\left\{e_{i j}(t)^{T} \Upsilon_{i j}^{1} e_{i j}(t)+e_{i j}(t-\tau(t))^{T} \Upsilon_{i j}^{2} e_{i j}(t-\tau(t))\right. \\
& \left.+e_{i j}\left(t-\tau_{c}(t)\right)^{T} \Upsilon_{i j}^{3} e_{i j}\left(t-\tau_{c}(t)\right)\right\} \\
=e & (t)^{T} \Upsilon^{1} e(t)+e(t-\tau(t))^{T} \Upsilon^{2} e(t-\tau(t))+e\left(t-\tau_{c}(t)\right)^{T} \Upsilon^{3} e\left(t-\tau_{c}(t)\right)
\end{aligned}
$$

Substituting Inequalities (12)-(18) into Equality (11), we have:

$$
\begin{aligned}
\mathcal{L} V(t, e(t))= & e^{T}(t)\left\{(-\eta+\alpha+\beta) I_{M N n}+I_{M} \otimes I_{N} \otimes(\Delta \Sigma)\right. \\
& \left.+\left(A^{d} \otimes I_{M}+I_{N} \otimes A^{r}-\Xi \otimes I_{N}^{1}\right) \otimes(P \Sigma)+\frac{1}{2} \Upsilon_{1}\right\} e(t) \\
+ & e^{T}(t-\tau(t))\left\{\zeta I_{M N n}+\frac{1}{2} \Upsilon_{2}\right\} e(t-\tau(t)) \\
+ & e^{T}\left(t-\tau_{c}(t)\right)\left\{\left(\frac{1}{\beta}\left(B^{d}\right)^{T}\left(B^{d}\right) \otimes I_{M}+\frac{1}{\alpha} I_{N} \otimes\left(B^{r}\right)^{T}\left(B^{r}\right)\right)\right. \\
& \left.\otimes(P \Sigma)^{T}(P \Sigma)+\frac{1}{2} \Upsilon_{3}\right\} e\left(t-\tau_{c}(t)\right)
\end{aligned}
$$

By Equation (10), we get:

$$
\mathcal{L} V(t) \leq-a V(t)+b V(t-\tau(t))+c V\left(t-\tau_{c}(t)\right) .
$$

Define:

$$
W(t)=e^{\gamma t} V(t)
$$


where constant $\gamma>0$ will be decided later, and use Equation (21) to compute the operator:

$$
\begin{aligned}
\mathcal{L} W(t) & =e^{\gamma t}[\gamma V(t)+\mathcal{L} V(t)] \\
& \leq e^{\gamma t}\left[\gamma V(t)-a V(t)+b V(t-\tau(t))+c V\left(t-\tau_{c}(t)\right)\right]
\end{aligned}
$$

which, after applying the generalized Itô's formula, gives:

$$
e^{\gamma t} \mathbb{E} V(t)=e^{\gamma t_{0}} \mathbb{E} V\left(t_{0}\right)+\mathbb{E} \int_{t_{0}}^{t} \mathcal{L} W(s) d s
$$

for any $t>t_{0}=0$. Hence, we have:

$$
\begin{aligned}
e^{\gamma t} \mathbb{E} V(t) \leq & \sup _{-\tilde{\tau} \leq s \leq 0} \mathbb{E} V(s)+\mathbb{E} \int_{0}^{t} e^{\gamma s}\{\gamma V(s)-a V(s) \\
& \left.+b V(s-\tau(s))+c V\left(s-\tau_{c}(s)\right)\right\} d s \\
\leq & \sup _{-\tilde{\tau} \leq s \leq 0} \mathbb{E} V(s)+(\gamma-a) \int_{0}^{t} e^{\gamma s} \mathbb{E} V(s) d s \\
& +b e^{\gamma \tau} \int_{0}^{t} e^{\gamma(s-\tau(s))} \mathbb{E} V(s-\tau(s)) d s \\
& +c e^{\gamma \tau_{c}} \int_{0}^{t} e^{\gamma\left(s-\tau_{c}(s)\right)} \mathbb{E} V\left(s-\tau_{c}(s)\right) d s
\end{aligned}
$$

which, by using the change of variables $s-\tau(s)=u$, gives:

$$
\begin{aligned}
\int_{0}^{t} e^{\gamma(s-\tau(s))} \mathbb{E} V(s-\tau(s)) d s & =\int_{-\tau(0)}^{t-\tau(t)} e^{\gamma u} \mathbb{E} V(u) \frac{d u}{1-\dot{\tau}(t)} \\
& \leq \frac{\tau}{1-\bar{\tau}} \sup _{-\check{\tau} \leq s \leq 0} \mathbb{E} V(s)+\frac{1}{1-\bar{\tau}} \int_{0}^{t} e^{\gamma u} \mathbb{E} V(u) d u
\end{aligned}
$$

and a further change of variables $s-\tau_{c}(s)=u$ gives:

$$
\begin{aligned}
\int_{0}^{t} e^{s-\tau_{c}(s)} \mathbb{E} V\left(s-\tau_{c}(s)\right) d s & =\int_{-\tau_{c}(0)}^{t-\tau_{c}(t)} e^{\gamma u} \mathbb{E} V(u) \frac{d u}{1-\dot{\tau}_{c}(t)} \\
& \leq \frac{\tau_{c}}{1-\bar{\tau}_{c}} \sup _{-\tilde{\tau} \leq s \leq 0} \mathbb{E} V(s)+\frac{1}{1-\bar{\tau}_{c}} \int_{0}^{t} e^{\gamma u} \mathbb{E} V(u) d u
\end{aligned}
$$

Substituting Inequalities (23) and (24) into Equality (22), we have:

$$
\begin{aligned}
e^{\gamma t} \mathbb{E} V(t) \leq & \left(1+\frac{b \tau}{1-\bar{\tau}} e^{\gamma \tau}+\frac{c \tau_{c}}{1-\bar{\tau}_{c}} e^{\gamma \tau_{c}}\right) \sup _{-\bar{\tau} \leq s \leq 0} \mathbb{E} V(s) \\
& +\left(\gamma-a+\frac{b}{1-\bar{\tau}} e^{\gamma \tau}+\frac{c}{1-\bar{\tau}_{c}} e^{\gamma \tau_{c}}\right) \int_{0}^{t} e^{\gamma s} \mathbb{E} V(s) d s
\end{aligned}
$$

By Condition (9), this equation:

$$
\gamma-a+\frac{b}{1-\bar{\tau}} e^{\gamma \tau}+\frac{c}{1-\bar{\tau}_{c}} e^{\gamma \tau_{c}}=0
$$


has at least one positive root. Let $\gamma$ be the smallest positive root of Equation (25). Therefore, we obtain:

$$
\mathbb{E} V(t) \leq\left(1+\frac{b \tau}{1-\bar{\tau}} e^{\gamma \tau}+\frac{c \tau_{c}}{1-\bar{\tau}_{c}} e^{\gamma \tau_{c}}\right) \sup _{-\bar{\tau} \leq s \leq 0} \mathbb{E} V(s) e^{-\gamma t}
$$

so that:

$$
\mathbb{E}\|e(t)\|^{2} \leq \frac{2}{\hat{p}}\left(1+\frac{b \tau}{1-\bar{\tau}} e^{\gamma \tau}+\frac{c \tau_{c}}{1-\bar{\tau}_{c}} e^{\gamma \tau_{c}}\right) \sup _{-\tilde{\tau} \leq s \leq 0} \mathbb{E} V(s) e^{-\gamma t},
$$

where $\hat{p}=\min \left\{p_{1}, p_{2}, \ldots, p_{n}\right\}$. The proof is hence complete.

Remark 3. Inequality (9) relies on the coupled matrices, the dynamics system, the noise strength and the time delays. The linear matrix inequality method is inadequate for assessing the stability of the synchronous solution.

When the time-varying delays are constant (i.e., $\tau(t)=\tau, \tau_{c}(t)=\tau_{c}$ ), we obtain the following corollary:

Corollary 1. Let Assumptions $\mathrm{H} 1$ and $\mathrm{H} 2$ be true, and let $f \in \operatorname{QUAD}(P, \Delta, \eta, \zeta)$. If there exist $\tau(t)$ and $\tau_{c}(t)$, such that:

$$
b+c-a<0,
$$

where:

$$
\begin{aligned}
a & =\lambda_{\min }\left\{(\eta-\alpha-\beta) I_{M N n}-I_{M} \otimes I_{N} \otimes(\Delta \Sigma)-\left(A^{d} \otimes I_{M}+I_{N} \otimes A^{r}-\Xi \otimes I_{N}^{1}\right)^{S} \otimes(P \Sigma)-\frac{1}{2} \Upsilon_{1}\right\}, \\
b & =\lambda_{\max }\left\{\zeta I_{M N n}+\frac{1}{2} \Upsilon_{2}\right\}, \\
c & =\lambda_{\max }\left\{\left(\frac{1}{\beta}\left(B^{d}\right)^{T}\left(B^{d}\right) \otimes I_{M}+\frac{1}{\alpha} I_{N} \otimes\left(B^{r}\right)^{T}\left(B^{r}\right)\right) \otimes(P \Sigma)^{T}(P \Sigma)+\frac{1}{2} \Upsilon_{3}\right\},
\end{aligned}
$$

then the solutions $e_{i j}(t)(i=1,2, \ldots, M, j=1,2, \ldots, N)$ of System (8) are globally and exponentially stable in the mean square.

When $\sigma_{i}(\cdot)=0$, we can get the following corollary:

Corollary 2. Let Assumptions $\mathrm{H} 1$ and $\mathrm{H} 2$ be true, and let $f \in \operatorname{QUAD}(P, \Delta, \eta, \zeta)$. If there exist $\tau(t)$ and $\tau_{c}(t)$, such that:

$$
0 \leq \check{\bar{\tau}} \leq 1-\frac{\zeta+c}{a}, \quad \text { and } \quad a>0
$$

where:

$$
\begin{aligned}
& a=\lambda_{\min }\left\{\eta I_{M N n}-I_{M} \otimes I_{N} \otimes(\Delta \Sigma)-\left(A^{d} \otimes I_{M}+I_{N} \otimes A^{r}-\Xi \otimes I_{N}^{1}\right)^{S} \otimes(P \Sigma)\right\}, \\
& c=\lambda_{\max }\left\{\left(\frac{1}{\beta}\left(B^{d}\right)^{T}\left(B^{d}\right) \otimes I_{M}+\frac{1}{\alpha} I_{N} \otimes\left(B^{r}\right)^{T}\left(B^{r}\right)\right) \otimes(P \Sigma)^{T}(P \Sigma)\right\},
\end{aligned}
$$

then the solutions $e_{i j}(t)(i=1,2, \ldots, M, j=1,2, \ldots, N)$ of System (8) are globally and exponentially stable in the mean square.

When $A^{d}=A^{r}=A, B^{d}=B^{r}=B$ and $\sigma_{i}(\cdot)=0$, we can get the following corollary: 
Corollary 3. Let Assumptions $\mathrm{H} 1$ and $\mathrm{H} 2$ be true, and let $f \in \operatorname{QUAD}(P, \Delta, \eta, \zeta)$. If there exist $\tau(t)$ and $\tau_{c}(t)$, such that:

$$
0 \leq \check{\tau} \leq 1-\frac{\zeta+c}{a}, \quad \text { and } \quad a>0
$$

where:

$$
\begin{aligned}
a & =\lambda_{\min }\left\{\eta I_{M N n}-I_{M} \otimes I_{N} \otimes(\Delta \Sigma)-\left(A \otimes I_{M}+I_{N} \otimes A-\Xi \otimes I_{N}^{1}\right)^{S} \otimes(P \Sigma)\right\}, \\
c & =\lambda_{\max }\left\{\left(\frac{1}{\beta} B^{T} B \otimes I_{M}+\frac{1}{\alpha} I_{N} \otimes B^{T} B\right) \otimes(P \Sigma)^{T}(P \Sigma)\right\},
\end{aligned}
$$

then the solutions $e_{i j}(t)(i=1,2, \ldots, M, j=1,2, \ldots, N)$ of System (8) are globally and exponentially stable in the mean square.

\section{Numerical Simulation}

In this section, we present some numerical simulation results that validate the theorem of the previous section.

Consider the 2D chaotic delayed neural network:

$$
\dot{s}(t)=f(t, s(t), s(t-\tau(t)))=-C s(t)+D \tanh (s(t))+E \tanh (s(t-\tau(t))),
$$

where $\tau(t)=1, C=\left[\begin{array}{ll}1 & 0 \\ 0 & 1\end{array}\right], D=\left[\begin{array}{cc}2 & -0.1 \\ -5 & 4.5\end{array}\right]$ and $E=\left[\begin{array}{cc}-1.5 & -0.1 \\ -0.2 & -4\end{array}\right]$.

Taking $P=\operatorname{diag}\{1,1\}$ and $\Delta=\operatorname{diag}\{5,11.5\}$, we have $\eta=0.15$ and $\zeta=3.25$, so that Condition (3) is satisfied [21]. Thus:

$$
\begin{aligned}
d x_{i}(t)= & \left\{f\left(t, x_{i}(t), x_{i}(t-\tau(t))\right)+\sum_{j=1}^{7} a_{i k}^{r} \Sigma x_{k}(t)+\sum_{k=1}^{7} b_{i k}^{r} \Sigma x_{k}\left(t-\tau_{c}(t)\right)-\varepsilon_{i} \Sigma\left(x_{i}(t)-y_{1}(t)\right)\right\} d t \\
& +\sigma_{i}\left(t, x_{i}(t), x_{i}(t-\tau(t)), x_{i}\left(t-\tau_{c}(t)\right)\right) d w(t), \quad i=1,2, \ldots, 7
\end{aligned}
$$

and:

$$
\begin{aligned}
d y_{i}(t)= & \left\{f\left(t, y_{j}(t), y_{j}(t-\tau(t))\right)+\sum_{s=1}^{5} a_{j s}^{r} \Sigma y_{s}(t)+\sum_{s=1}^{5} b_{j s}^{r} \Sigma y_{s}\left(t-\tau_{c}(t)\right)\right\} d t \\
& +\sigma_{j}\left(t, y_{j}(t), y_{j}(t-\tau(t)), y_{j}\left(t-\tau_{c}(t)\right)\right) d w(t), \quad j=1,2, \ldots, 5,
\end{aligned}
$$


where:

$$
\begin{aligned}
& A^{r}=203.5999\left[\begin{array}{ccccc}
-4 & 1 & 1 & 1 & 1 \\
1 & -4 & 1 & 1 & 1 \\
1 & 1 & -3 & 1 & 0 \\
1 & 1 & 1 & -4 & 1 \\
1 & 1 & 0 & 1 & -3
\end{array}\right] \\
& B^{r}=\left[\begin{array}{ccccc}
-4 & 1 & 1 & 1 & 1 \\
1 & -3 & 1 & 0 & 1 \\
1 & 1 & -3 & 1 & 0 \\
1 & 0 & 1 & -2 & 0 \\
1 & 1 & 0 & 0 & -2
\end{array}\right] \\
& A^{d}=212.8970\left[\begin{array}{ccccccc}
-6 & 1 & 1 & 1 & 1 & 1 & 1 \\
1 & -3 & 1 & 1 & 0 & 0 & 0 \\
1 & 1 & -5 & 1 & 1 & 1 & 0 \\
1 & 1 & 1 & -6 & 1 & 1 & 1 \\
1 & 0 & 1 & 1 & -4 & 0 & 1 \\
1 & 0 & 1 & 1 & 0 & -3 & 0 \\
1 & 0 & 0 & 1 & 1 & 0 & -3
\end{array}\right] \\
& B^{d}=\left[\begin{array}{ccccccc}
-3 & 1 & 1 & 0 & 1 & 0 & 0 \\
1 & -5 & 1 & 1 & 1 & 1 & 0 \\
1 & 1 & -4 & 1 & 0 & 0 & 1 \\
0 & 1 & 1 & -2 & 0 & 0 & 0 \\
1 & 1 & 0 & 0 & -4 & 1 & 1 \\
0 & 1 & 0 & 0 & 1 & -2 & 0 \\
0 & 0 & 1 & 0 & 1 & 0 & -2
\end{array}\right]
\end{aligned}
$$

$\Xi=718.4148 \operatorname{diag}\{1,1,0,0,0\}, \tau_{c}(t)=0.01 \frac{e^{t}}{1+e^{t}}, \sigma_{i}\left(t, x_{i}(t), x_{i}(t-\tau(t)), x_{i}\left(t-\tau_{c}(t)\right)\right)=$ $0.01 \operatorname{diag}\left\{x_{i 1}(t)+x_{i 1}(t-\tau(t))+x_{i 1}\left(t-\tau_{c}(t)\right), x_{i 2}(t)+x_{i 2}(t-\tau(t))+x_{i 2}\left(t-\tau_{c}(t)\right)\right\}$ and $\sigma_{i}\left(t, y_{i}(t)\right.$, $\left.y_{i}(t-\tau(t)), y_{i}\left(t-\tau_{c}(t)\right)\right)=0.01 \operatorname{diag}\left\{y_{i 1}(t)+y_{i 1}(t-\tau(t))+y_{i 1}\left(t-\tau_{c}(t)\right), y_{i 2}(t)+y_{i 2}(t-\tau(t))+\right.$ $\left.y_{i 2}\left(t-\tau_{c}(t)\right)\right\}$.

Computations then yield $\tau=1, \bar{\tau}=0, \tau_{c}=0.01, \bar{\tau}_{c}=0.0025, \Upsilon_{i j}=0.01 I_{2}$ for $i=1,2, \ldots, M$, and $j=1,2$. Then, the solutions of Equalities (10) are: $a=5.40, b=3.26, c=2.0334$; after solving Equation (25), we obtain $\gamma=0.016$, so that the conditions of Theorem 1 are satisfied.

The initial conditions for this simulation are $x_{i j}\left(t_{0}\right)=-(2 i+k) \cos \left(t_{0}\right), i=1,2, \ldots, 7$, $y_{j s}\left(t_{0}\right)=-(2(i+7)+s) \cos \left(t_{0}\right), \quad j=1,2, \ldots, 5, \quad k=1,2$ and for all $t_{0} \in[-1,0]$. The numerical approximation to the solution of stochastic differential Equations (27) and (28) are found by the Euler-Maruyama method [44] with $\Delta t=0.001$, and the trajectories of the pinning control gains are shown in Figures 1-3. Figure 4 shows the time evolution of the synchronization errors without pinning control. One can see that the pinning control directly affects the synchronization of two complex network, which cannot be reached without pinning control. 


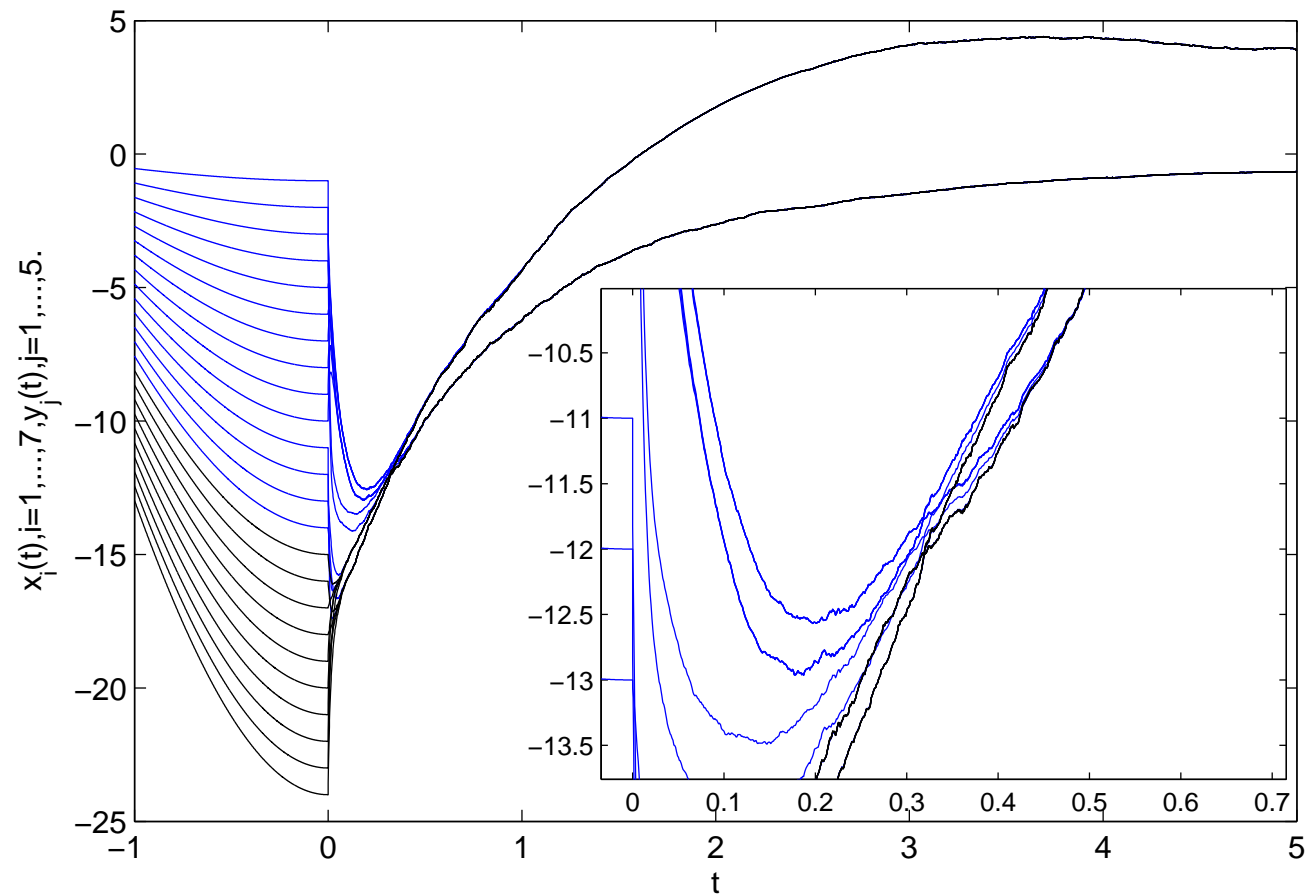

Figure 1. The trajectories of the state variables of $x_{i}$ and $y_{j}(i=1,2, \ldots, 7$ and $j=1, \ldots, 5)$ in Systems (27) and (28) under pinning control. The blue lines show the evolution of the driver systems, and the black lines represent the trajectory of the driver systems in the figure, as they do for the following figures.

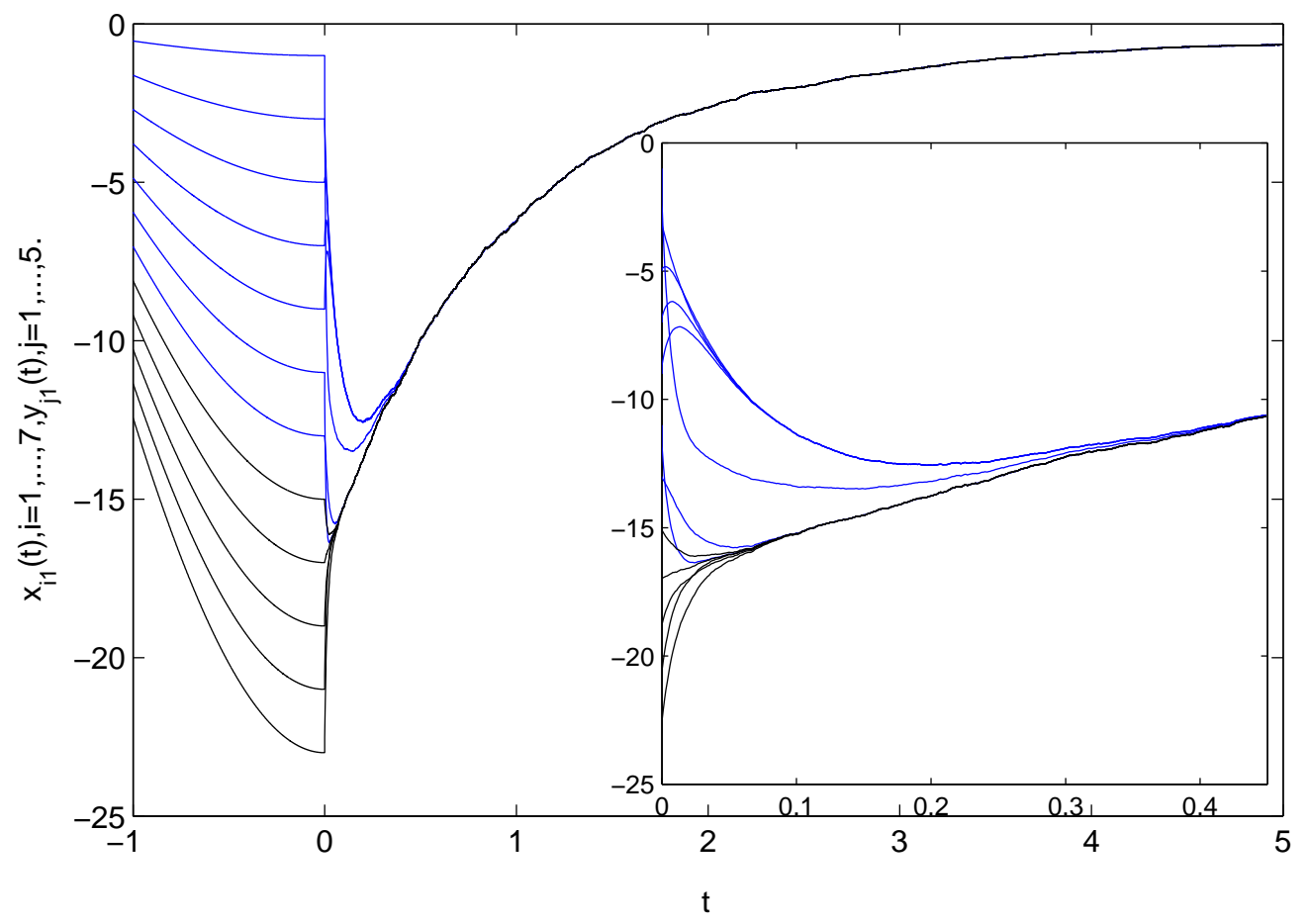

Figure 2. The time evolution of $x_{i 2}$ and $y_{j 2}(i=1,2, \ldots, 7$ and $j=1, \ldots, 5)$ in Systems (27) and (28) under pinning control. 


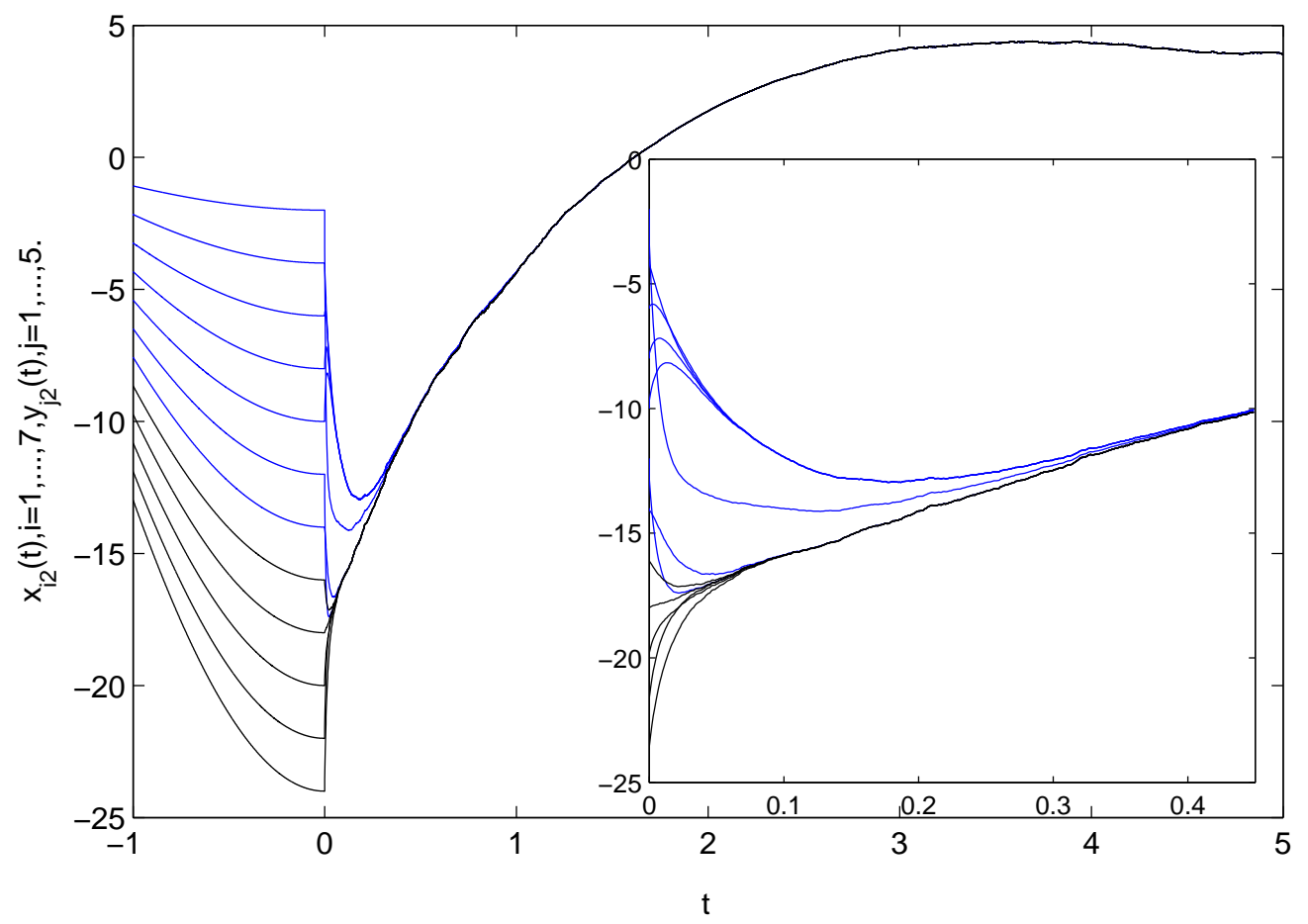

Figure 3. The time evolution of $x_{i 2}$ and $y_{j 2}(i=1,2, \ldots, 7$ and $j=1, \ldots, 5)$ in Systems (27) and (28) under pinning control.

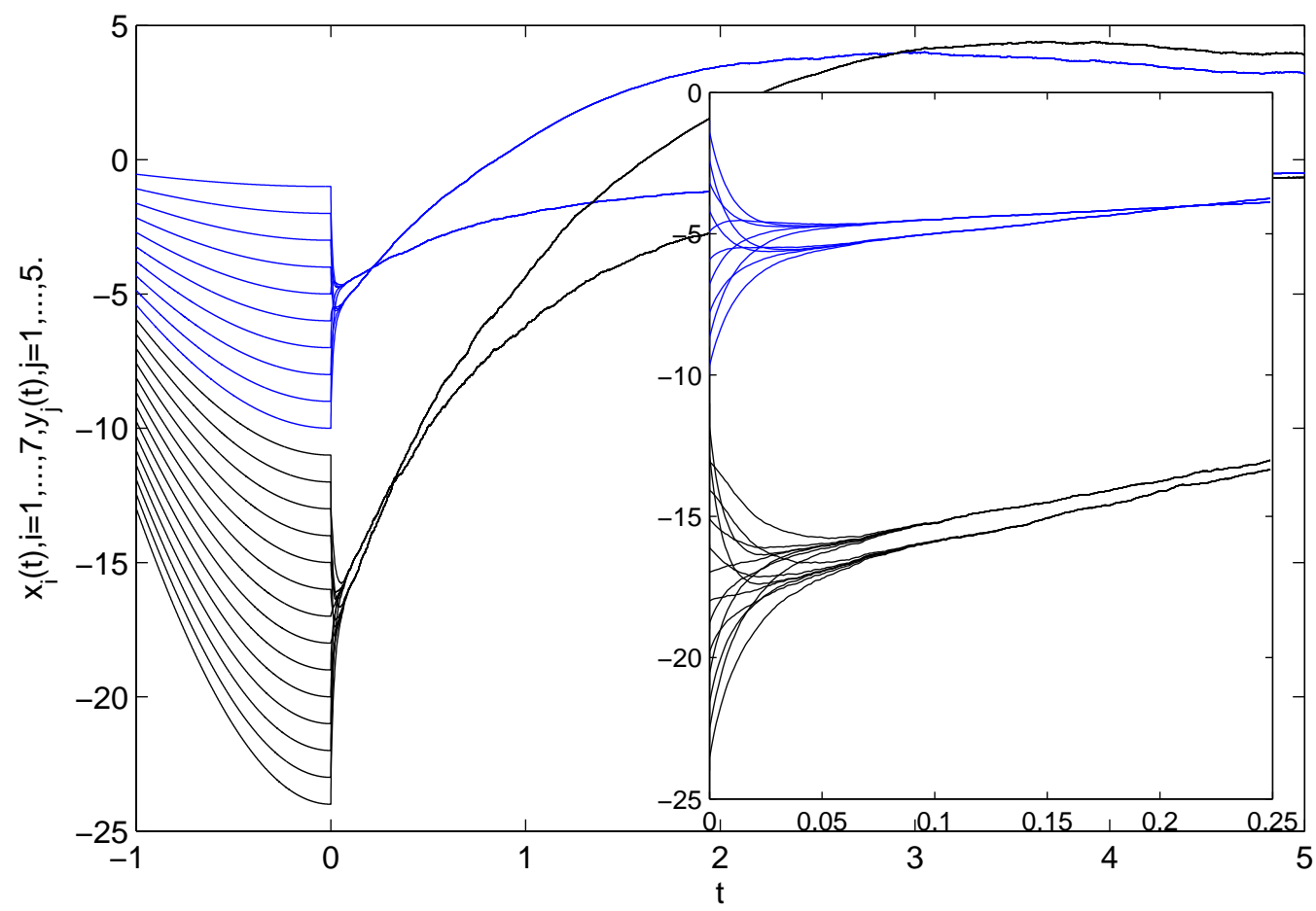

Figure 4. The time evolution of $x_{i}$ and $y_{j}(i=1,2, \ldots, 5)$ in Systems (27) and (28) without pinning control. 


\section{Conclusions}

In this paper, we investigated the synchronization of two different stochastic complex networks with hybrid coupling and time-varying delay. Specifically, we achieved global exponential synchronization in the mean square by applying a pinning control scheme to a small fraction of the nodes and derived sufficient conditions for the global exponential stability of synchronization in the mean square. In addition, we considered some numerical examples that have verified the effectiveness of the proposed techniques. In the future, we will consider the analysis of pinning-controlled networks with switching topologies and drive-response delays.

\section{Acknowledgments}

This work was supported by the Shenzhen Basic Research Project (JCYJ20130331152625792). The author is very grateful to the reviewers and the editor for their valuable comments and suggestions to improve the presentation of the paper.

\section{Conflicts of Interest}

The author declares that there is no conflict of interest regarding the publication of this paper.

\section{References}

1. Girvan, M.; Newman, M.E. Community structure in social and biological networks. Proc. Natl. Acad. Sci. USA 2002, 99, 7821-7826.

2. Arenas, A.; Diaz-Guilera, A.; Kurths, J.; Moreno, Y.; Zhou, C. Synchronization in complex networks. Phys. Rep. 2008, 469, 93-153.

3. Dye, C.; Williams, B.G. The population dynamics and control of tuberculosis. Science 2010 , 328, 856-861.

4. Dorfler, F.; Bullo, F. Synchronization and transient stability in power networks and nonuniform Kuramoto oscillators. SIAM J. Control Optim. 2012, 50, 1616-1642.

5. Dörfler, F.; Chertkov, M.; Bullo, F. Synchronization in complex oscillator networks and smart grids. Proc. Natl. Acad. Sci. USA 2013, 110, 2005-2010.

6. Zhou, J.; Zhang, H.; Xiang, L.; Wu, Q. Synchronization of coupled harmonic oscillators with local instantaneous interaction. Automatica 2012, 48, 1715-1721.

7. Boccaletti, S.; Kurths, J.; Osipov, G.; Valladares, D.L.; Zhou, C.S. The synchronization of chaotic systems. Phys. Rep. 2002, 366, 1-101.

8. Pecora, L.M.; Carroll, T.L. Synchronization in chaotic systems. Phys. Rev. Lett. 1990, 64, 821-824.

9. Tönjes, R.; Blasius, B. Perturbation analysis of complete synchronization in networks of phase oscillators. Phys. Rev. E 2009, 80, doi: 10.1103/PhysRevE.80.026202 .

10. Lu, J.; Ho, D.W.C. Globally exponential synchronization and synchronizability for general dynamical networks. IEEE Trans. Syst. Man Cybern. 2010, 40, 350-361. 
11. Yang, X.; Cao, J.; Lu, J. Synchronization of coupled neural networks with random coupling strengths and mixed probabilistic time-varying delays. Int. J. Robust Nonlinear Control 2013, 23, 2060-2081.

12. Belykh, V.N.; Belykh, I.V.; Mosekilde, E. Cluster synchronization modes in an ensemble of coupled chaotic oscillators. Phys. Rev. E 2001, 63, doi:10.1103/PhysRevE.63.036216.

13. Wang, J.; Feng, J.; Xu, C.; Zhao, Y. Cluster synchronization of nonlinearly-coupled complex networks with nonidentical nodes and asymmetrical coupling matrix. Nonlinear Dyn. 2012, 67, 1635-1646.

14. Rosenblum, M.G.; Pikovsky, A.S.; Kurths, J. Phase Synchronization of Chaotic Oscillators. Phys. Rev. Lett. 1996, 76, 1804-1807.

15. Van Vreeswijk, C. Partial synchronization in populations of pulse-coupled oscillators. Phys. Rev. 1996, E54, 5522-5537.

16. Zhou, P.; Bai, R.; Zheng, J. Projective synchronization for a class of fractional-order chaotic systems with fractional-order in the (1,2) Interval. Entropy 2015, 17, 1123-1134.

17. Zhou, X.; Xiong, L.; Cai, X. Adaptive switched generalized function projective synchronization between two hyperchaotic systems with unknown parameters. Entropy 2014, 16, 377-388.

18. Chen, T.; Liu, X.; Lu, W. Pinning complex networks by a single controller. IEEE Trans. Circuits Syst. I Regul. Pap. 2007, 54, 1317-1326.

19. Zhao, J.; Lu, J.; Wu, X. Pinning control of general complex dynamical networks with optimization. Sci. China Inf. Sci. 2010, 53, 813-822.

20. Lu, J. ; Cao, J. ; Ho, D.W.C. Adaptive stabilization and synchronization for chaotic Lur'e systems with time-varying delay. IEEE Trans. Circuits Syst. I Regul. Pap. 2008, 55, 1347-1356.

21. Yang, X.; Cao, J. Adaptive pinning synchronization of complex networks with stochastic perturbations. Discrete Dyn. Nat. Soc. 2010, 2010, doi.org/10.1155/2010/416182.

22. Ma, W.; Li, C.; Wu, Y. Adaptive synchronization of fractional neural networks with unknown parameters and time delays. Entropy 2014, 16, 6286-6299.

23. Wang, J.; Feng, J.; Xu, C.; Zhao, Y. Exponential synchronization of stochastic perturbed complex networks with time-varying delays via periodically intermittent pinning. Commun. Nonlin. Sci. Numer. Simul. 2013, 18, 3146-3157.

24. Haeri, M.; Dehghani, M. Impulsive synchronization of Chen's hyperchaotic system. Phys. Lett. A 2006, 356, 226-230.

25. Dai, A.; Zhou, W.; Feng, J.; Fang, J.; Xu, S. Exponential synchronization of the coupling delayed switching complex dynamical networks via impulsive control. Adv. Differ. Equ. 2013, 2013, doi:10.1186/1687-1847-2013-195.

26. Ahmadlou, M.; Adeli, H. Fuzzy synchronization likelihood with application to attention-deficit/hyperactivity disorder. Clin. EEG Neurosci. 2011, 42, 6-13.

27. Yang, X.; Cao, J.; Lu, J. Stochastic synchronization of complex networks with nonidentical nodes via hybrid adaptive and impulsive control. IEEE Trans. Circuits Syst. I Regul. Pap. 2012, 59, 371-384. 
28. Razminia, A.; Majd, V.J.; Baleanu, D. Chaotic incommensurate fractional order Rössler system: Active control and synchronization. Adv. Differ. Equ. 2011, 2011, doi:10.1186/ 1687-1847-2011-15.

29. $\mathrm{Wu}, \mathrm{Y}$;; Liu, L. Exponential outer synchronization between two uncertain time-varying complex networks with nonlinear coupling. Entropy 2015, 17, 3097-3109.

30. Li, C.; Sun, W.; Kurths, J. Synchronization between two coupled complex networks. Phys. Rev. E 2007, 76, doi:10.1103/PhysRevE.76.046204.

31. Li, C.; Xu, C.; Sun, W.; Xu, J.; Kurths, J. Outer synchronization of coupled discrete-time networks. Chaos Interdiscip. J. Nonlin. Sci. 2009, 19, doi:10.1063/1.3068357.

32. Wu, X.; Zheng, W.X.; Zhou, J. Generalized outer synchronization between complex dynamical networks. Chaos Interdiscip. J. Nonlin. Sci. 2009, 19, doi: 10.1063/1.3072787.

33. Wang, G.; Cao, J.; Lu, J. Outer synchronization between two nonidentical networks with circumstance noise. Physica A 2010, 389, 1480-1488.

34. Cao, J.; Wang, Z.; Sun, Y. Synchronization in an array of linearly stochastically coupled networks with time delays. Physica A 2007, 385, 718-728.

35. Li, C.; Chen, G. Synchronization in general complex dynamical networks with coupling delays. Physica A 2004, 343, 263-278.

36. Lu, W.; Chen, T.; Chen, G. Synchronization analysis of linearly coupled systems described by differential equations with a coupling delay. Physica D 2006, 221, 118-134.

37. He, W.; Cao, J. Exponential synchronization of hybrid coupled networks with delayed coupling. IEEE Trans. Neural Netw. 2010, 21, 571-583.

38. Cao, J.; Wang, Z.; Sun, Y. Synchronization in an array of linearly stochastically coupled networks with time delays. Physica A 2007, 385, 718-728.

39. Ding, X.; Gao, Y.; Zhou, W.; Tong, D.; Su, H. Adaptive almost surely asymptotically synchronization for stochastic delayed neural networks with Markovian switching. Adv. Differ. Equ. 2013, 2013, 1-12.

40. Wang, J.; Xu, C.; Feng, J.; Kwong, M.K.; Austin, F. Mean-square exponential synchronization of markovian switching stochastic complex networks with time-varying delays by pinning control. Abstr. Appl. Anal. 2012, 2012, doi: 10.1155/2012/298095.

41. Lu, J.; Ho, D.W.C.; Wu, L. Exponential stabilization of switched stochastic dynamical networks. Nonlinearity 2009, 22, 889-911.

42. Mao, X. Stability of stochastic differential equations with Markovian switching. Stoch. Process Their Appl. 1999, 79, 45-67.

43. Yuan, C.; Mao, X. Robust stability and controllability of stochastic differential delay equations with Markovian switching. Automatica 2004, 40, 343-354.

44. Higham, D.J. An algorithmic introduction to numerical simulation of stochastic differential equations. SIAM Rev. 2001, 43, 525-546.

(c) 2015 by the author; licensee MDPI, Basel, Switzerland. This article is an open access article distributed under the terms and conditions of the Creative Commons Attribution license (http://creativecommons.org/licenses/by/4.0/). 